\title{
Genotypes and phenotypes of a family with a deaf child carrying combined heterozygous mutations in SLC26A4 and GJB3 genes
}

\author{
YUNLONG LI ${ }^{1,2}$ and BAOSHENG ZHU ${ }^{1,2}$ \\ ${ }^{1}$ Medical Faculty, Affiliated Hospital of Kunming University of Science and Technology \\ (The First People's Hospital of Yunnan Province), Kunming University of Science and Technology, \\ Kunming, Yunan 650500; ${ }^{2}$ Genetic Diagnosis Center, The First People's Hospital of Yunnan Province, \\ Kunming, Yunnan 650032, P.R. China
}

Received May 11, 2015; Accepted March 29, 2016

DOI: $10.3892 / \mathrm{mmr} .2016 .5280$

\begin{abstract}
Mutations in the SLC26A4 gene have been shown to cause a type of deafness referred to as large vestibular aqueduct syndrome (LVAS), whereas mutations in the GJB3 gene have been associated with nonsyndromic deafness. However, the clinical phenotypes of these mutations vary and remain to be fully elucidated. The present study performed genetic analysis of a Chinese family, in which the child was deaf and the parents were healthy. Sanger sequencing demonstrated that the affected individual harbored three heterogeneous mutations in the SLC26A4 and GJB3 genes, as follows: SLC26A4 IVS-2 A>G, SLC26A4 c.2168 A>G and GJB3 c.538 C>T. The affected individual exhibited hearing loss and was diagnosed with LVAS by computed tomography scan. The mother and father of the affected individual harbored the heterogeneous mutations of SLC26A4 IVS-2 $\mathrm{A}>\mathrm{G}$ and GJB3 c.538 $\mathrm{C}>\mathrm{T}$, and the heterozygous mutation of SLC26A4 c.2168 A>G, respectively. Neither parents exhibited any hearing loss. The results obtained from the deaf patient provided genetic and clinical evidence that carrying combined heterogeneous mutations in the GJB3 and SLC26A4 genes may be involved in the etiology of severe hearing loss, of which the mechanism requires further examination.
\end{abstract}

Correspondence to: Dr Yunlong Li, Medical Faculty, Affiliated Hospital of Kunming University of Science and Technology (The First People's Hospital of Yunnan Province), Kunming University of Science and Technology, 727 Jing Ming Nan Road, Kunming, Yunnan 650500, P.R. China

E-mail: yunlongli.km@gmail.com

Professor Baosheng Zhu, Genetic Diagnosis Center, The First People's Hospital of Yunnan Province, 157 Jinbi Road, Kunming, Yunnan 650032, P.R. China

E-mail: bszhu@aliyun.com

Key words: mutations, nonsyndromic deafness, large vestibular aqueduct syndrome

\section{Introduction}

Deafness is the partial or total inability to hear (1). In adults, it causes problems in communication (2). In children, as well as problems in communication, deafness affects the development of language. Several factors may cause deafness $(3,4)$, including genetic mutations, noise exposure, illness, toxic chemicals and aging. A substantial proportion of cases of deafness are caused by genetic mutations (4-7). Genetic deafness can be divided into two categories: Syndromic and nonsyndromic deafness. The clinical phenotypes of genetic deafness remain to be fully elucidated and the same mutation may result in different clinical phenotypes in different individuals.

Mutations in genes, including the GJB3 (8), GJB2 (9), SLC26A4 (10) and 12S RNA genes (11), have been previously associated with genetic deafness. Large vestibular aqueduct syndrome (LVAS) is one type of genetic deafness that can be caused by genetic mutations in the SLC26A4 gene (12). Conversely, mutations in the GJB3 gene have been associated with nonsyndromic deafness (DFNA2B), which is inherited in an autosomal dominant pattern $(13,14)$. The clinical phenotypes, including age of onset and the hearing loss severity, of mutations in the GJB3 gene vary. Whether there is a correlation between genetic deafness and mutations in the GJB3 and SLC26A4 genes remains to be elucidated.

In the present study, mutations in the GJB3, GJB2, SLC26A4 and 12S RNA genes were screened in a family with genetic deafness. The screening identified mutations in the GJB3 and SLC26A4 genes of the deaf patient, from which the relative clinical information were examined and discussed. The results of the present study may enable the establishment of genetic counseling specific to deaf patients harboring mutations in the GJB3 and SLC26A4 genes.

\section{Materials and methods}

Patients and genomic DNA isolation. Whole blood samples were obtained from the study family at the First People's Hospital of Yunnan Province (Kunming, China) during their visit to the clinical center on 13th August 2013. The affected individual exhibited hearing loss, whereas neither the father nor the mother of the affected child showed signs of hearing 


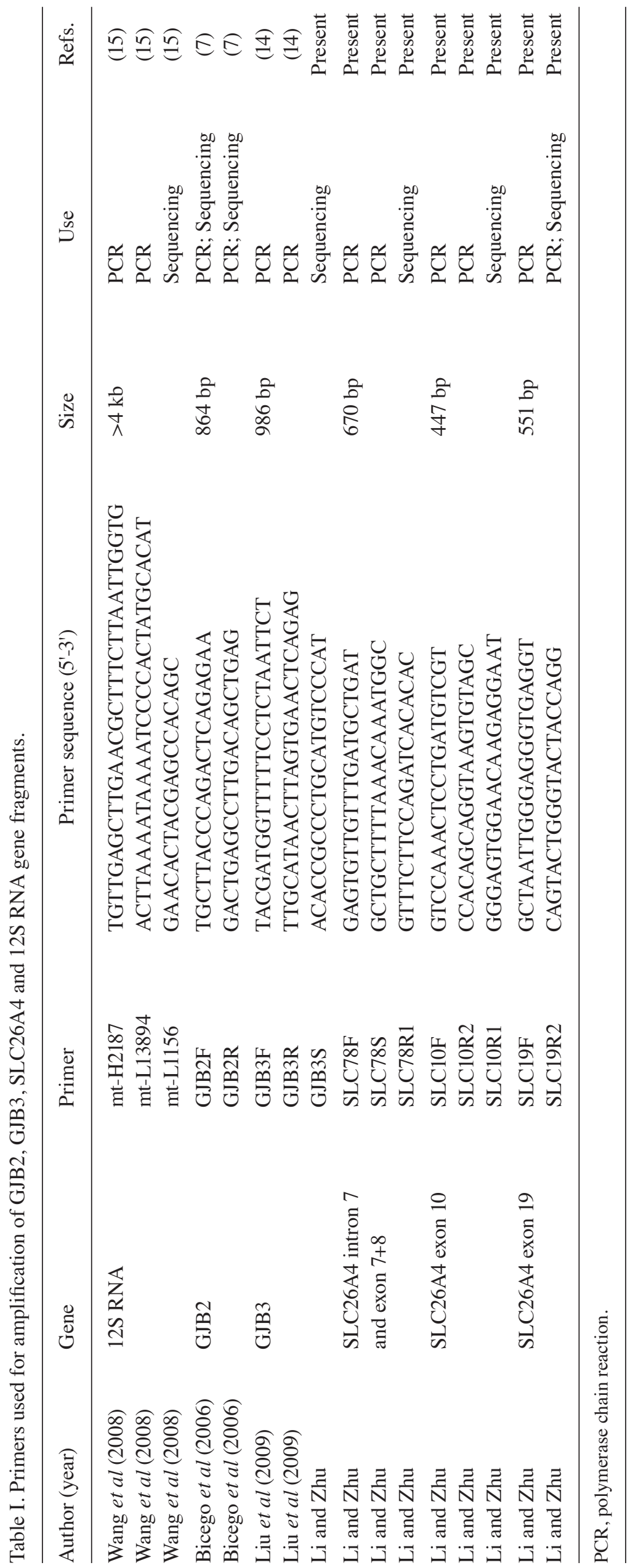



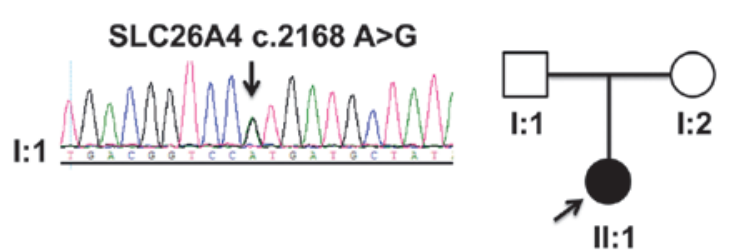

GJB3 c.538 C>T

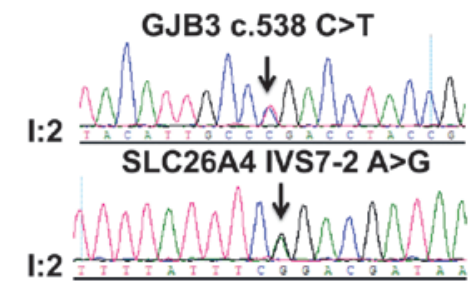

$\mathrm{I}: 2$

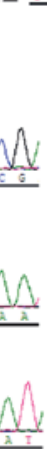

Figure 1. Sequencing results of a family with a deaf child (II:1, dark cycle) carrying heterozygous mutations in the SLC26A4 and GJB3 genes. Genomic DNA was isolated from whole blood samples and used for mutation screening. I:1 and I:2 represent the father and mother of the affected child, respectively.

loss or impairment. The present study was approved by the Ethics Committee of the First People's Hospital of Yunnan Province (Affiliated Hospital of Kunming University of Science and Technology). Written informed consent was signed and obtained from the participants. Genomic DNA from the whole blood samples were extracted using a DNA extraction kit (cat. no. ZTLYQ-F50; Xi'an Tianlong Science \& Technology Co., Ltd., China), according to the manufacturer's protocol.

Polymerase chain reaction (PCR) amplification and sequencing. The GJB2, GJB3, SLC26A4 and 12S rRNA gene fragments were amplified using PCR. The primers used for amplification and sequencing of the fragments are shown in Table I and were obtained from the Beijing Genomics Institute (Shenzhen, China). The amplification of the fragments in the $12 \mathrm{~S}$ rRNA gene referred to the descriptions by Wang et al (15), and was performed in a $25 \mu 1$ reaction mixture containing 10X LA PCR Buffer II ( $\mathrm{Mg}^{2+}$ Plus), 2.5 units of Takara LA Taq (Takara Biotechnology Co., Ltd., Dalian, China), $400 \mu \mathrm{M}$ of each dNTP, $0.2 \mu \mathrm{M}$ of each primer and $50 \mathrm{ng}$ DNA. The following conditions were used for PCR amplification: Denaturation cycle at $94^{\circ} \mathrm{C}$ for $1 \mathrm{~min}$, followed by 30 cycles of denaturation at $94^{\circ} \mathrm{C}$ for $30 \mathrm{sec}$ and $65.6^{\circ} \mathrm{C}$ for $5 \mathrm{~min}$, and ended with a final extension at $72^{\circ} \mathrm{C}$ for $10 \mathrm{~min}$. Amplification of the GJB2, GJB3 and SLC26A4 gene fragments was performed in a volume of $25 \mu \mathrm{l}$ containing $30 \mathrm{ng}$ genomic DNA, $50 \mu \mathrm{M}$ dNTP, 10X LA TaqTM PCR buffer, 2.5 units of Takara LA TaqTM and $0.2 \mu \mathrm{M}$ of each forward and reverse primer. The PCR amplification for these gene fragments followed a denaturation cycle of $94^{\circ} \mathrm{C}$ for $5 \mathrm{~min}$, followed by 35 cycles of denaturation at $94^{\circ} \mathrm{C}$ for $30 \mathrm{sec}$, annealing at $55^{\circ} \mathrm{C}$ for SLC26A4 exon10, $58^{\circ} \mathrm{C}$ for GJB3, $59^{\circ} \mathrm{C}$ for SLC26A4 exon $19,63^{\circ} \mathrm{C}$ for SLC26A4 intron7 and exon7+8, or $60^{\circ} \mathrm{C}$ for GJB2, and extension at $72^{\circ} \mathrm{C}$ for $30 \mathrm{sec}$ for SLC26A4 exon10, $35 \mathrm{sec}$ for SLC26A4 exon 19, $40 \mathrm{sec}$ for SLC26A4 intron 7 and exon 7+8, $55 \mathrm{sec}$ for GJB3 or $60 \mathrm{sec}$ for GJB2, and ended with a final extension step at $72^{\circ} \mathrm{C}$ for 7 min.

The PCR products were purified using a Genomic DNA Purification kit (cat. no. DP204-02; Tiangen Biotech Co., Ltd., Tiangen, China) and were sequenced using the sequencing primers (Table I) and Big Dye Terminator v.3.1 Cycle Sequencing kit (cat. no. 4337456; Applied Biosystems; Thermo Fisher Scientific, Inc., Waltham, MA, USA) on an ABI Prism 3730 DNA sequencer (Applied Biosystems; Thermo Fisher Scientific, Inc.).

Evolutionary conservation analysis. Evolutionary conservation analysis was performed by aligning amino acid sequences of the GJB3 and SLC26A4 proteins of eight vertebrate species from the GenBank database (http://www. ncbi.nlm.nih.gov/genbank/), as follows: Homo sapiens (GJB3, GenBank accession no. NP_001005752.1; SLC26A4 GenBank accession no. NP_000432.1), Macaca mulatta (GJB3, GenBank accession no. XP_001108150.1;SLC26A4 GenBank accession no. XP_001094049.1), Canis familiaris (GJB3, GenBank accession no. XP_005629030.1;SLC26A4 GenBank accession no. XP_005631003.1), Bos taurus (GJB3, GenBank accession no. NP_001098465.1;SLC26A4 GenBank accession no, XP_002686849.2), Mus musculus (GJB3, GenBank accession no. NP_001153484.1;SLC26A4 GenBank accessio no. NP_035997.1), Rattus norvegicus (GJB3, GenBank accession no. NP_062113.1;SLC26A4 GenBank accession no. NP_062087.1), Gallus gallus (GJB3, GenBank accession no.XP_004947838.1; SLC26A4 GenBank accession no. XP_425419.3), and Danio rerio (GJB3, GenBank accession no. NP_001017685.1; SLC26A4 GenBank accession no. NP_001159387.1).

\section{Results and Discussion}

In the present study, the $12 \mathrm{~S}$ rRNA gene, exons $7,8,10$ and 19, and intron 7 of the SLC26A4 gene, and the entire 
A

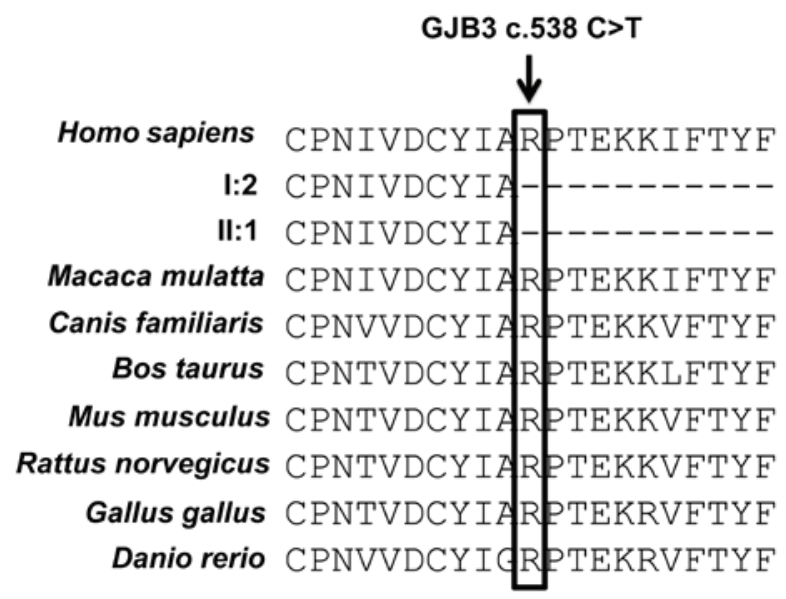

B

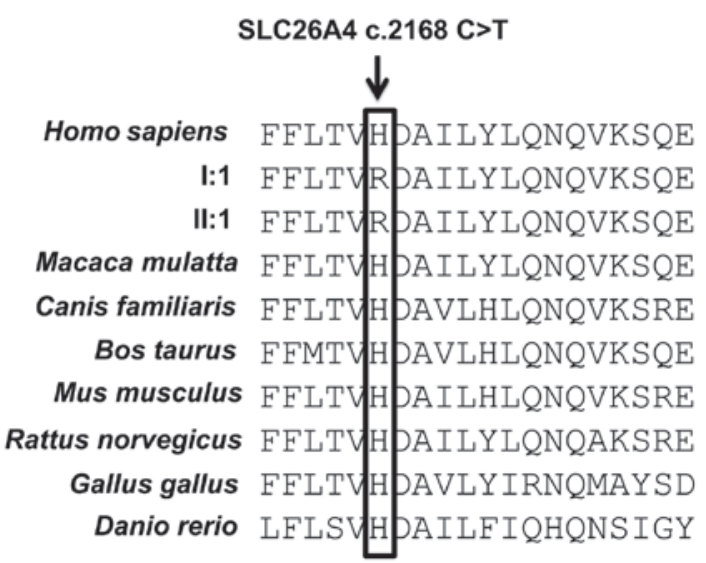

C
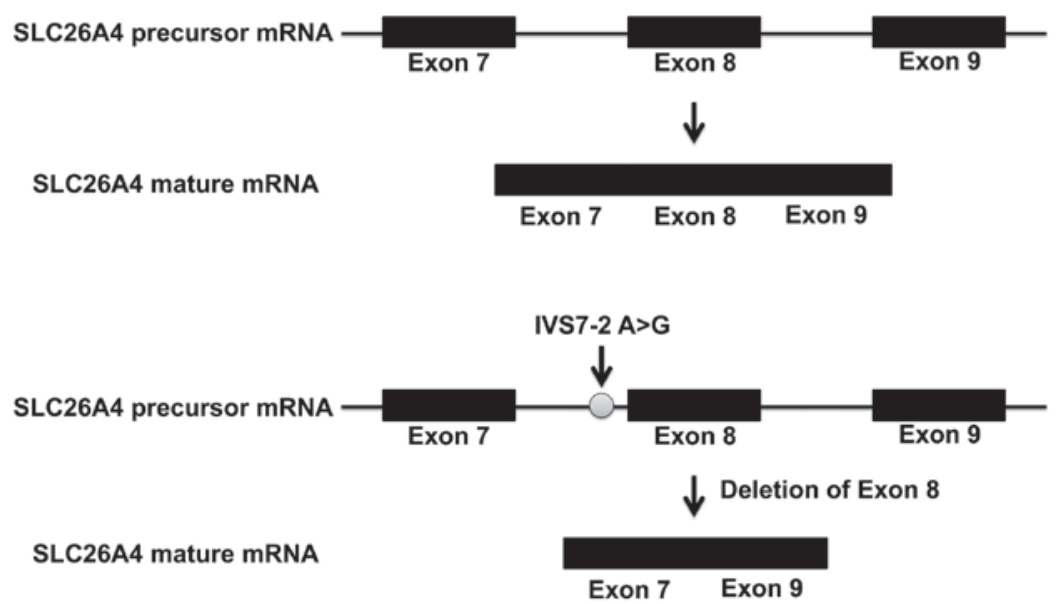

Figure 2. (A) Evolutionary analysis of GJB3 codon R180 in different vertebrate species. Sequences for the vertebrate species were retrieved from the GenBank database. (B) Evolutionary analysis of the SLC26A4 codon H723 in different vertebrate species. Sequences for vertebrate species were retrieved from the GenBank database. (C) Compared with normal splicing of the SLC26A4 precursor mRNA, the IVS7-2 A $>$ G splicing site mutation resulted in aberrant splicing of exon 8 in the SLC26A4 precursor mRNA. I:1, father of the affected individual; I:2, mother of the affected individual; II:1, affected individual.

coding region of the GJB3 and GJB2 genes, were examined by direct sequencing in a Chinese family comprising a child with LVAS (Fig. 1). The family was from the Yunnan province of southwestern China. Chromosome examination of the affected individual (subject II:1) showed no chromosome aberration (data not shown). The heterozygous mutations of the GJB3 and SLC26A4 genes in the family were identified (Fig. 1). The affected individual (subject II:1) harbored the following heterozygous mutations of SLC26A4: IVS7-2 A $>\mathrm{G}$ and c.2168 C>T, and the GJB3 mutation, c.538 C>T. The age at onset of deafness was 4 years old. The mutations confirmed the clinical diagnosis following a computed tomography scan, since LVAS has been reported to be caused by compound mutations in SLC26A4 (IVS7-2 A > G and c.2168 C > T) $(10,12)$. The mother of the affected individual (subject I:2) showed no hearing loss, but harbored heterozygous mutations of SLC26A4 (IVS7-2 A>G) and GJB3 (c.538 C>T). The GJB3 c. $538 \mathrm{C}>\mathrm{T}$ mutation has been shown to be correlated with a form of nonsyndromic deafness, termed DFNA2B, which is inherited in an autosomal dominant pattern $(13,14)$. The time of onset and clinical phenotype of deafness caused by the GJB3 c.538 C>T mutation varies in different individuals, 
and this may explain why subject I:2, who harbored the GJB3 c.538 C>T mutation, did not exhibit any hearing loss. The father of the affected individual (subject I:1) harbored heterozygous mutations of SLC26A4 (c.2168 C>T) and did not exhibit any hearing loss. These findings are consistent with reports that heterozygous mutations of SLC26A4 c.2168 $\mathrm{C}>\mathrm{T}$ alone are unable to cause deafness.

In addition to the three mutations described above, no other common mutations associated with inheritable nonsyndromic deafness were identified in the fragments sequenced in the present study, including c.35delG, c.167delT, c.176_191del16, c.235delC and c.299_300delAT in the GJB2 gene; c.547 G>A in the GJB3 gene; IVS7-2 A>G, c.1174 A>T, c.1226 G>A, c.1229 C>T and c.2162 C>T in the SLC26A4 gene, and m.1494 $\mathrm{C}>\mathrm{T}$ and $\mathrm{m} .1555 \mathrm{~A}>\mathrm{G}$ in the $12 \mathrm{~S}$ rRNA gene.

The present study also found a number of reported genetic variants of the GJB3 and GJB2 genes in the family examined, which were listed in the dbSNP database (http://www.ncbi.nlm.nih.gov/projects/SNP/). These variants included rs41310442 in the GJB3 gene (subject I:1, CC; subject I:2, CT; subject II:1, CT) and rs138547875 in the GJB2 gene (subject I:1. AG; subject I:2, GG; subject II:1, $\mathrm{AG})$. The SNPs did not result in the alteration of amino acids. The m.1736 A>G mutation in the 16S rRNA gene was found in subject I:1, whereas A was identified at this position in the codon of subjects I:1 and II:1.

Evolutionary conservation analysis showed that GJB3 180R and SLC26A4 723H were highly conserved in different vertebrate species (Fig. 2A and B). These results indicated that mutations in GJB3 180R and SLC26A4 723H may result in significant functional changes of these proteins. The splicing site mutation SLC26A4 IVS7-2 A>G can alter the normal splicing of SLC26A4 precursor mRNA (Fig. 2C). SLC26A4 IVS7-2 A>G results in the deletion of exon 8 during splicing of the SLC26A4 precursor mRNA, as shown in Fig. 2C. Taken together, SLC26A4 IVS7-2 A>G and c.2168 $\mathrm{C}>\mathrm{T}$, and GJB3 c.538 C>T disrupt the normal function of SLC26A4 and GJB3 respectively.

In the present study, the affected individual with LVAS harbored three heterozygous mutations of SLC26A4 IVS7-2 A>G, SLC26A4 c.2168 C>T and GJB3 c.538 C>T. LVAS can be caused by the combined heterozygous mutations of SLC26A4 IVS7-2 A>G and c.2168 C>T. Whether the GJB3 c.538 $\mathrm{C}>\mathrm{T}$ mutation can increase or decrease the hearing loss caused by SLC26A4 IVS7-2 A $>\mathrm{G}$ and c.2168 C>T mutations remains to be elucidated. The affected individual (subject II:1) was examined at the onset time by multiple auditory steady-state evoked responses (ASRR), otoacoustic emission (OAE) and auditory brainstem response (ABR) assessments. In the ASRR test, the right ear passed at 500 and 1,000 $\mathrm{HZ}$, and the auditory thresholds were 80 and $120 \mathrm{dBHL}$ respectively. The right ear showed no response to 2,000 and 4,000 HZ. The left ear only passed at 4,000 HZ, with an auditory threshold of $110 \mathrm{dBHL}$. The left ear showed no response to the tests at $500,1,000$ or $2,000 \mathrm{HZ}$. In the ABR assessment, a $95 \mathrm{dBHL}$ click evoked no ABR waveforms in the left ear and normal ABR waveforms in the right ear. The auditory threshold of the right ear was $85 \mathrm{dBHL}$. Neither the right or left ear passed the transient OAE (TEOAE) or distortion product OAE assessments. These results indicated that the combined mutations in the SLC26A4 and GJB3 genes may have resulted in a severe hearing loss.

Deafness, or hearing loss, is one of the most common birth defects worldwide. Statistically, 1/500 newborns has bilateral permanent sensorineural hearing loss (>40 dBHL) (1). The exact pathologic mechanism of deafness remains to be fully elucidated. The GJB2, GJB3, SLC26A4 and 12S RNA genes are considered to be closely associated with inheritable deafness (16-18). By examining the nucleotide sequence of these genes in a Chinese family, a deaf patient carrying combined heterozygous mutations in the SLC26A4 and GJB3 genes was identified in the present study. The clinical data of this patient showed severe hearing loss.

Although patients with deafness have been shown to harbor the same mutations, the clinical phenotype of inheritable nonsydromic deafness varies $(14,19,20)$. The results of the present study indicated that combined heterozygous mutations of the SLC264 and GJB3 genes may result in severe hearing loss. These results contribute to the understanding of clinical phenotype of deaf patients carrying combined mutations in the SLC26A4 and GJB3 genes. These conclusions require further confirmation in other patients carrying similar mutations. Further functional investigations are also required to reveal the molecular mechanism underlying deafness associated with these three heterozygous mutations in the SLC26A4 and GJB3 genes.

\section{Acknowledgements}

The present study was supported by the Education Commission of Yunnan Province (grant no. 2014Z036), the National Natural Science Foundation of China (grant nos. 81460424 and 31060155 ) and the Kunming University of Science and Technology (grant no. KKZ3201360025). The authors would like to thank the patients who participated in this study.

\section{References}

1. Lasak JM, Allen P, McVay $T$ and Lewis D: Hearing loss: Diagnosis and management. Prim Care 41: 19-31, 2014.

2. Oishi $\mathrm{N}$ and Schacht J: Emerging treatments for noise-induced hearing loss. Expert Opin Emerg Drugs 16: 235-245, 2011.

3. Roizen NJ: Nongenetic causes of hearing loss. Ment Retard Dev Disabil Res Rev 9: 120-127, 2003.

4. Willems PJ: Genetic causes of hearing loss. N Engl J Med 342: 1101-1109, 2000.

5. Levenson D: New testing guidelines for hearing loss support next-generation sequencing: Testing method may help determine genetic causes of hearing loss among patients whose phenotypes are not easily distinguished clinically. Am J Med Genet A 164A: vii-viii, 2014.

6. Wei Q, Wang S, Yao J, Lu Y, Chen Z, Xing G and Cao X: Genetic mutations of GJB2 and mitochondrial 12S rRNA in nonsyndromic hearing loss in Jiangsu Province of China. J Transl Med 11: 163, 2013.

7. Bicego M, Beltramello M, Melchionda S, Carella M, Piazza V, Zelante L, Bukauskas FF, Arslan E, Cama E, Pantano S, et al: Pathogenetic role of the deafness-related M34T mutation of Cx26. Hum Mol Genet 15: 2569-2587, 2006.

8. Frei K, Ramsebner R, Hamader G, Lucas T, Schoefer C, Baumgartner WD, Wachtler FJ and Kirschhofer K: Lack of association between Connexin 31 (GJB3) alterations and sensorineural deafness in Austria. Hear Res 194: 81-86, 2004. 
9. Kokotas H, Grigoriadou M, Korres GS, Ferekidou E, Giannoulia-Karantana A, Kandiloros D, Korres S and Petersen MB: Are GJB2 mutations an aggravating factor in the phenotypic expression of mitochondrial non-syndromic deafness? J Hum Genet 55: 265-269, 2010.

10. Smith RJ and Robin NH: Genetic testing for deafness-GJB2 and SLC26A4 as causes of deafness. J Commun Disord 35: 367-377, 2002.

11. Estivill X, Govea N, Barceló E, Badenas C, Romero E, Moral L, Scozzri R, D'Urbano L, Zeviani M and Torroni A: Familial progressive sensorineural deafness is mainly due to the mtDNA A $1555 \mathrm{G}$ mutation and is enhanced by treatment of aminoglycosides. Am J Hum Genet 62: 27-35, 1998.

12. Azaiez H, Yang T, Prasad S, Sorensen JL, Nishimura CJ, Kimberling WJ and Smith RJ: Genotype-phenotype correlations for SLC26A4-related deafness. Hum Genet 122: 451-457, 2007.

13. Oh SK, Choi SY, Yu SH, Lee KY, Hong JH, Hur SW, Kim SJ, Jeon CJ and Kim UK: Evaluation of the pathogenicity of GJB3 and GJB6 variants associated with nonsyndromic hearing loss. Biochim Biophys Acta 1832: 285-291, 2013.

14. Liu XZ, Yuan Y, Yan D, Ding EH, Ouyang XM, Fei Y, Tang W, Yuan H, Chang Q, Du LL, et al: Digenic inheritance of non-syndromic deafness caused by mutations at the gap junction proteins Cx26 and Cx31. Hum Genet 125: 53-62, 2009.
15. Wang HW, Jia X, Ji Y, Kong QP, Zhang Q, Yao YG and Zhang YP: Strikingly different penetrance of LHON in two Chinese families with primary mutation G11778A is independent of mtDNA haplogroup background and secondary mutation G13708A. Mutat Res 643: 48-53, 2008.

16. Huang S, Wang G, Jiang Y, Yuan Y, Han D, Song Y and Dai P: Phenotype and genotype of deaf patients with combined genomic and mitochondrial inheritance models. Mitochondrion 13: 791-794, 2013

17. Sagong B, Baek JI, Oh SK, Na KJ, Bae JW, Choi SY, Jeong JY, Choi JY, Lee SH, Lee KY and Kim UK: A rapid method for simultaneous screening of multi-gene mutations associated with hearing loss in the Korean population. PLoS One 8: e57237, 2013.

18. Du W, Cheng J, Ding H, Jiang Z, Guo Y and Yuan H: A rapid method for simultaneous multi-gene mutation screening in children with nonsyndromic hearing loss. Genomics 104: 264-270, 2014.

19. Smith RJH, Shearer AE, Hildebrand MS and Van Camp G: Deafness and hereditary hearing loss overview. In: GeneReviews ${ }^{\circledR}$ (Internet). Pagon RA, Adam MP, Ardinger HH, WallaceSE, Amemiya A, Bean LJH, Bird TD, Fong CT, Mefford HC, Smith RJH and Stephens K (eds). University of Washington, Seattle, WA, pp1993-2016, 1993.

20. Bitner-Glindzicz M: Hereditary deafness and phenotyping in humans. Br Med Bull 63: 73-94, 2002. 